\title{
Optimization Control on 'Electricity-Hydrogen' Coordination in Active Distribution Network Part Two Case Study
}

\author{
Liang Wang ${ }^{1,}$, , Hua Zhang ${ }^{1, ~ b}$, Ming Tang ${ }^{2, ~ c}$, Bo Zhou, d and Gang Chen ${ }^{1, \mathrm{e}}$ \\ 1 State Grid Sichuan Electric Power Research Institute, Chengdu 610072, China \\ ${ }^{2}$ Energy Internet Research Institute of Tsinghua University, Chengdu 610072, China \\ ascuwl@sina.com, bzhanghua002@163.com, ctangming@tsinghua-eiri.org, dzbv_s@126.com, \\ egangchen08@gmail.com
}

Keywords: 'Electricity-Hydrogen' coordinated dispatch; electrolysis cell; efficiency model.

Abstract. In the first part of the paper, the models of electrolysis cell and AND were presented. Based on the models, the optimal model with the consideration of the network operation constraints in the distribution level is presented in this paper. Case studies were carried out to prove the advantages of the electrolysis cell.

\section{Introduction}

Hydrogen is a kind of energy storage material which is easy to storage. The electrolysis cell can realize large-scale and long-term energy storage based on two important energy carriers - electricity and hydrogen. Therefore, the consumptive capacity of renewable energy in ADN can be improved.

At present, the research level mainly remains on the micro-grid level, basically single point optimization, without consideration of network operation constraints. In this paper, the 'Electric-hydrogen' coordinated dispatch in distribution network is realized with the consideration of the network operation constraints, which has been presented in [1]

The rest of the paper is organized as follows: The second section presents optima model; The third section shows the results of case study and the final section draws the conclusions.

\section{Optimal Model}

The models of the electrolysis cell and AND including reaction principle, efficiency model, electric module, gas module, operation constraints of AND and dispatch model were presented in [1].

The objective function is:

$$
\min \sum c(t) \times\left|P_{T}(t)\right| \Delta t+a(t) \times n_{T}(t)
$$

Where $c, P_{T}, a, n_{T}$ are electric cost, purchased electric energy, gas cost and purchased gas energy. The constraints are presented in [1]

To convert the nonlinear constraints into linear constraints, piecewise linearization method is used and integer variables are added[2,3].

$$
\begin{aligned}
& P_{E L} \eta=\sum_{i}\left(a_{i} P_{E L i}+b_{i} \delta_{E L i}\right) \\
& P_{E L i, \min } \delta_{E L i} \leq P_{E L i} \leq P_{E L i, \max } \delta_{E L i} \\
& P_{E L}=\sum_{i} P_{E L i} \\
& \delta_{E L}=\sum_{i} \delta_{E L i}
\end{aligned}
$$




\section{Case Study}

In this paper, the IEEE 33-node radial distribution case system is selected and reference the case system in [4], the micro-turbine, wind turbine and the electrolysis cell are added to the ADN. The structure of the case system is shown in Fig. 1:

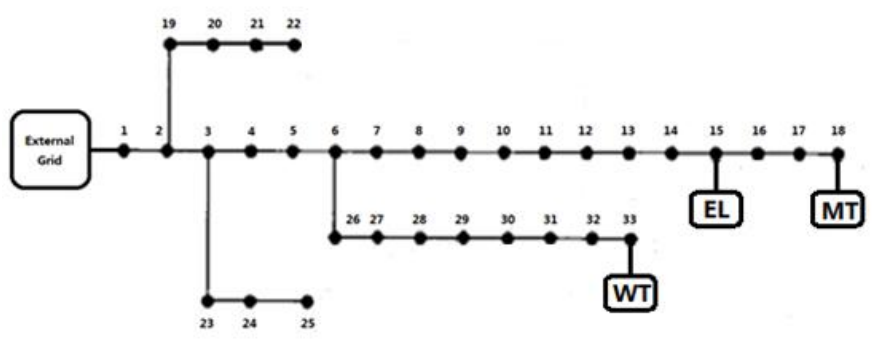

Fig. 1 Topology structure of case system

This system consists of 37 branches, 5 rings and radial operation. Voltage level is $12.66 \mathrm{kV}$, total maximum active load is $18.82 \mathrm{MW}$, minimum is $7.67 \mathrm{MW}$, total maximum reactive load is $11.66 \mathrm{Mvar}$, minimum is $4.76 \mathrm{Mvar}$, power factor is set to 0.85 . The basic configuration parameters are: at node 18 , there is a micro-turbine MT (MT18), installed capacity is $12 \mathrm{MW}$, power factor is set to 0.9; at node 33 , there is a wind turbine WT (WT33), installed capacity is $10 \mathrm{MW}$; at node 15 , there is an the electrolysis cell, rated power is $5 \mathrm{MW}$.

In this paper, MATLAB2015b, IBM ILOG CPLEX12.6 and Matpower5.1 are used. Test platform is a dual-core CPU, $4 \mathrm{G}$ memory PC.

1) electricity load and heat load

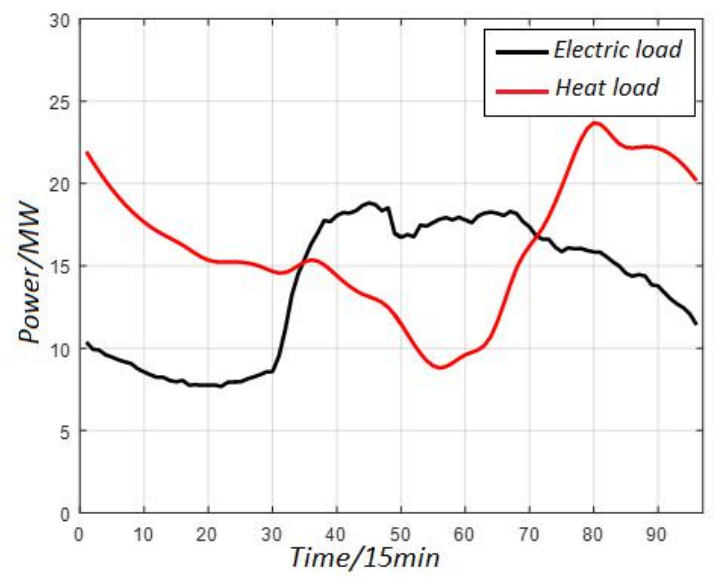

2) electricity price

Fig. 2 Case data- electricity load and heat load

Tab. 1 Case data- electricity price

\begin{tabular}{ccc}
\hline Period & time & price (yuan) \\
\hline Peak period & $11: 00-15: 00,19: 00-22: 00$ & 1.08 \\
Normal period & $8: 00-10: 00,16: 00-18: 00$ & 0.73 \\
Valley period & $1: 00-7: 00,23: 00-24: 00$ & 0.36 \\
\hline
\end{tabular}

3) wind power curve 


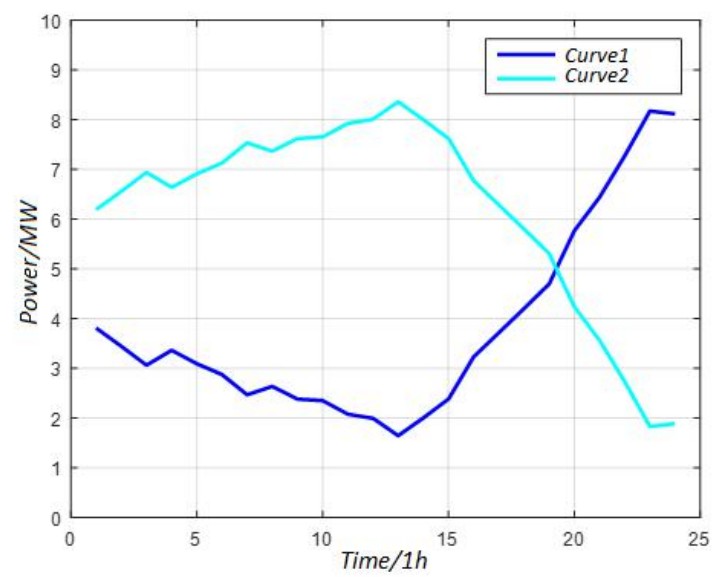

Fig. 3 Case data-wind power curve

Tab. 2 Dispatch Results of the case

Without the electrolysis cell

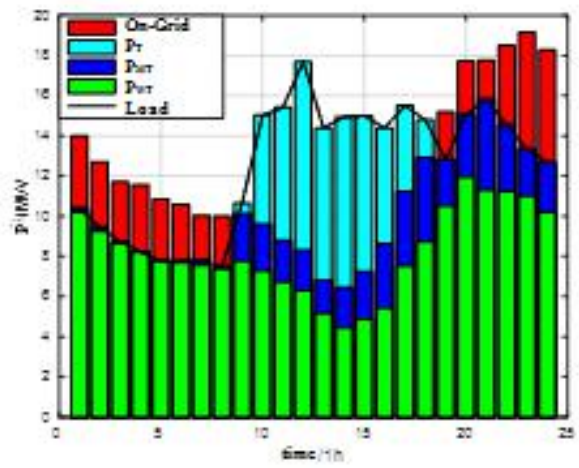

With the electrolysis cell

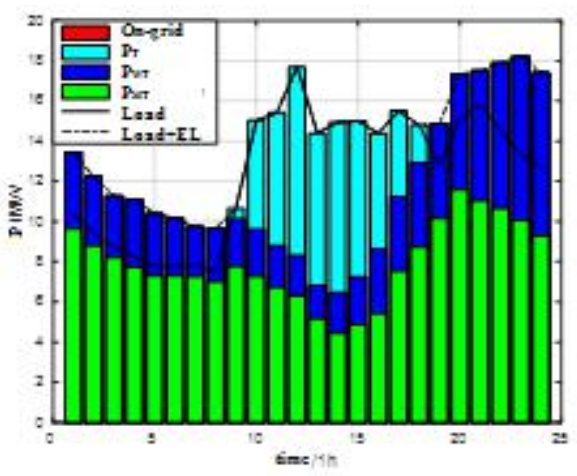

Gas module

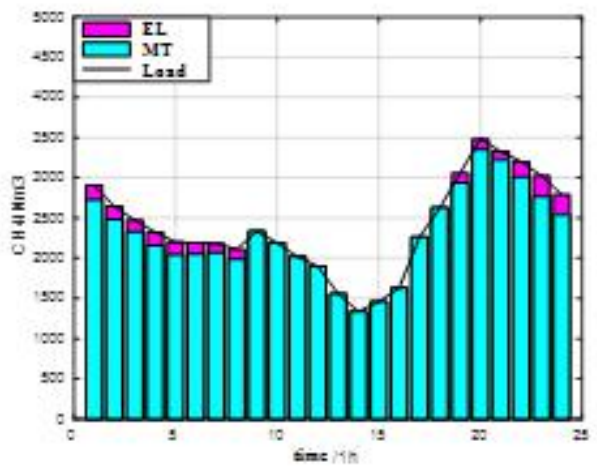

Tab. 3 Dispatch Results of the case

\begin{tabular}{c|c|c|c|c|c|c}
\hline $\begin{array}{c}\text { Installed } \\
\text { capacity }\end{array}$ & Curve & El & $\begin{array}{c}\text { Electricity } \\
\text { cost } \\
\text { (yuan })\end{array}$ & $\begin{array}{c}\text { Gas cost } \\
\text { (yuan })\end{array}$ & $\begin{array}{c}\text { Total cost } \\
\text { (yuan })\end{array}$ & $\begin{array}{c}\text { Change of } \\
\text { objective value }\end{array}$ \\
\hline 10 & 1 & No & $5.7695 \mathrm{e}+04$ & $1.7781 \mathrm{e}+05$ & $2.5006 \mathrm{e}+05$ & \multirow{2}{*}{$0.2596 \mathrm{e}+05$} \\
\cline { 2 - 5 } MW & Yes & $5.6079 \mathrm{e}+04$ & $1.6502 \mathrm{e}+05$ & $2.2110-\mathrm{e}+05$ & \\
\hline
\end{tabular}


It can be seen from Tab. 2-3, under the same wind power installed capacity and the same wind power output curve, the addition of the electrolysis cell can reduce the objective function cost.

The power curve of the electrolysis cell is shown in Fig. 4:

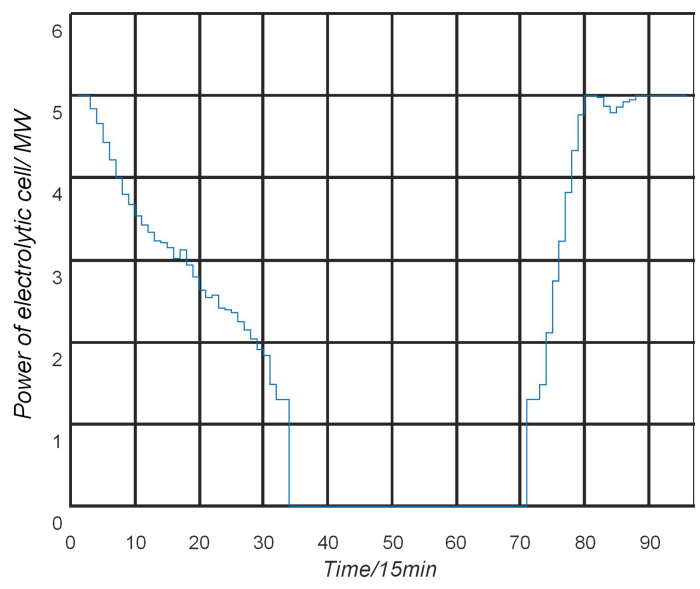

Fig. 4 Work power curve of the electrolysis cell

The work power and work time of the electrolysis cell mainly depend on the power and time of wind power injecting to the main network: In the electricity aspect, the electrolysis cell works as an electric load and does not cost using the redundant wind power, at the same time the electrolysis cell works as a gas source, the greater power and the longer time makes more hydrogen production, which can reduce the fuel cost of micro-turbine.

What's more, the highest and the lowest node voltage of the whole network are shown as Fig. 5-10.

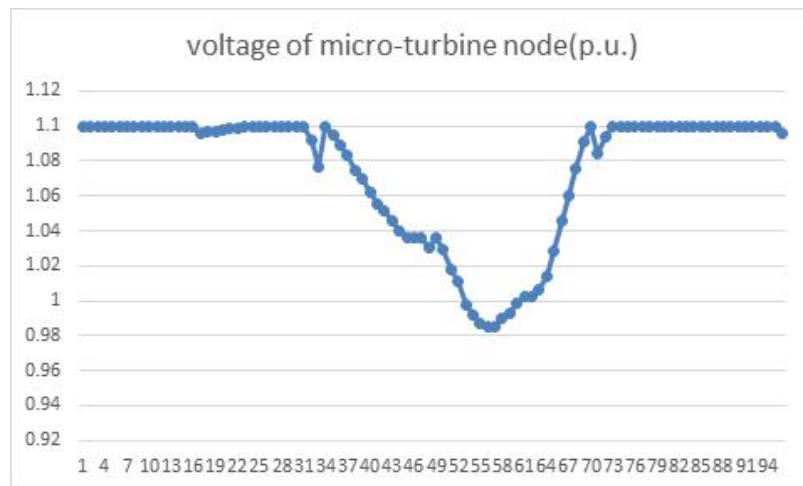

Fig. 5 The highest node voltage of the whole network (micro-turbine node)

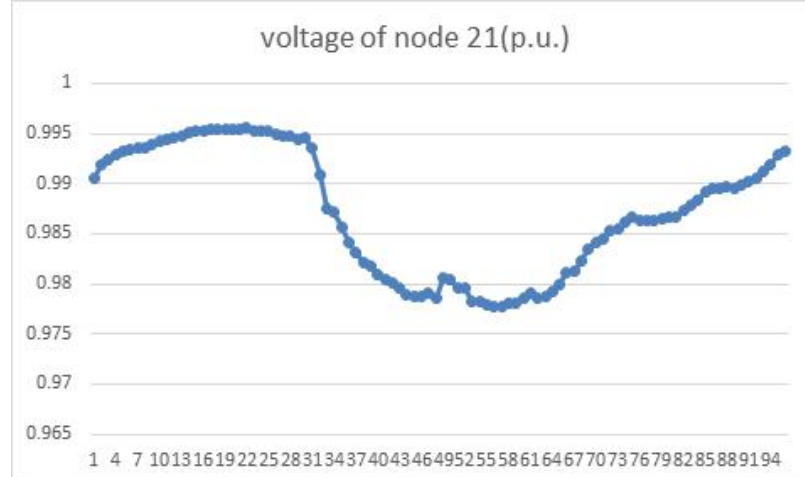

Fig. 6 The lowest node voltage of the whole network (node 21)

It can be seen from Fig. 5 that the node voltage reaches to the maximum at the micro-turbine node and the wind turbine node, and decreases gradually along the flow direction. 


\section{Conclusion}

This paper researches on the 'electric-hydrogen' coordinated dispatch in ADN basing on the proposed efficiency model of the electrolysis cell. The results of the case study show that the addition of the electrolysis cell increases the consumptive capacity of wind power and reduces the fuel demand of the micro-turbine at the same time. What's more, with the integration of the electrolysis cell, the node voltage of the micro-turbine can be avoided to exceed the upper voltage limit by the reduction of the power flow in the branch.

\section{Acknowledgements}

This work was supported by Science and Technology Project of State Grid Sichuan Electric Power Company (52199716002Q).

\section{References}

[1] Gang Chen, Bo Zhou, Lan Hou, et al. Optimization Control on 'Electricity-Hydrogen' Coordination in Active Distribution Network Part One System Modeling [C], 2017 International Conference on Advances in Energy and Environmental Science(ICAEES 2017), Shenzhen, China.

[2] Xiong $\mathrm{Wu}$, Xiuli Wang, Jianxue Wang, et al. Economic generation scheduling of a microgrid using mixed integer programming[J]. Proceedings of the CSEE, 2013, 28(33): 1-8.

[3] Miguel Carrión, José M. Arroyo. A Computationally Efficient Mixed-Integer Linear Formulation for thermal Unit Commitment Problem[J]. IEEE Transactions on Power Systems, 2006, 3(21): 1371-1378.

[4] Yibing Liu, Wenchuan Wu, Boming Zhang, et al. A mix integer second-ordercone programming based active and reactive power coordinated multi-period optimization for active distribution network [J]. Proceedings of the CSEE, 2014, 16(34): 2575 - 2583. 